Document downloaded from:

http://hdl.handle.net/10251/150431

This paper must be cited as:

Lago, MA.; Rupérez Moreno, MJ.; Martínez Martínez, F.; Martinez-Sanchis, S.; Bakic, P.; Monserrat, C. (2015). Methodology based on genetic heuristics for in-vivo characterizing the patient-specific biomechanical behavior of the breast tissues. Expert Systems with Applications. 42(21):7942-7950. https://doi.org/10.1016/j.eswa.2015.05.058

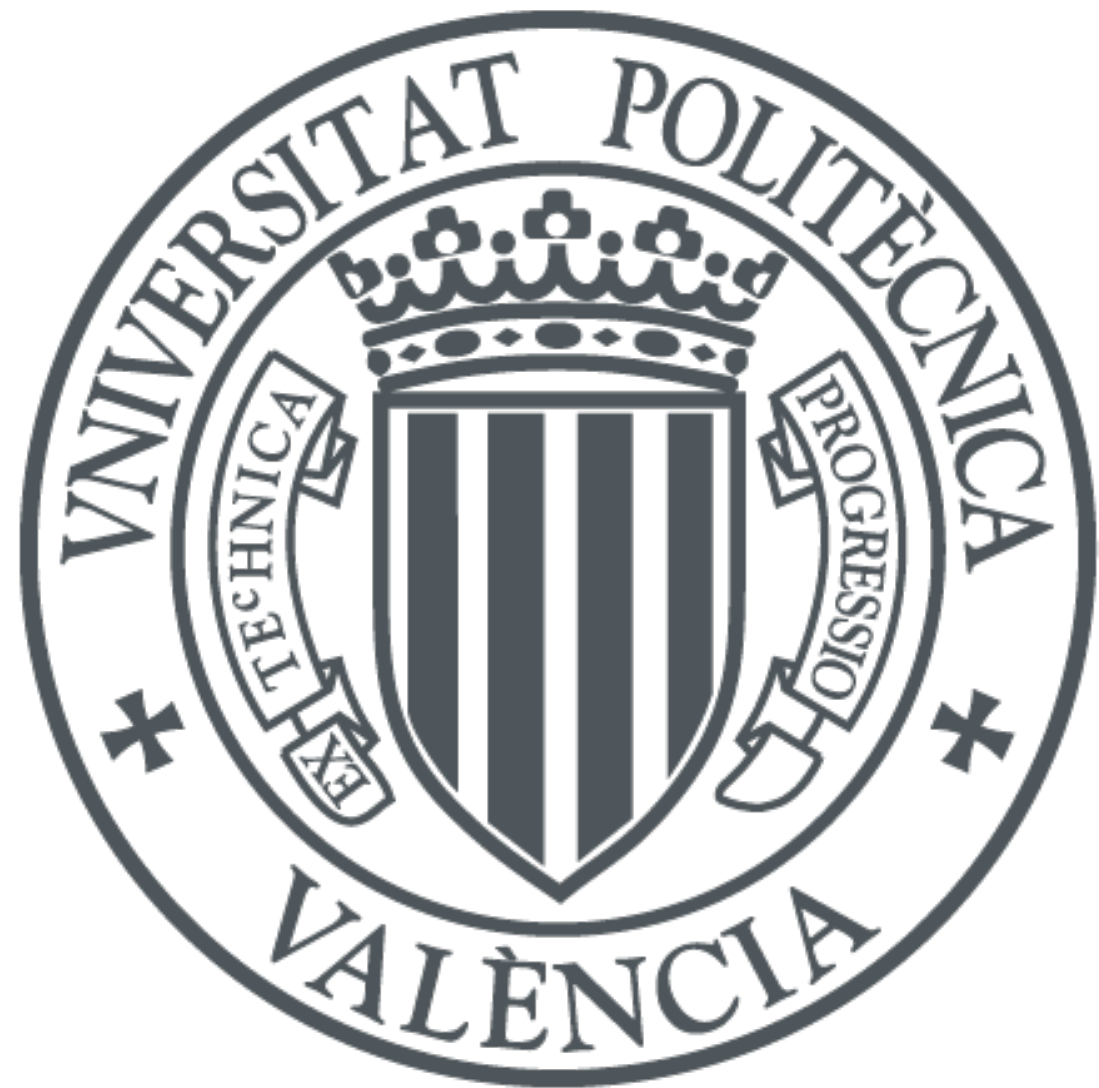

The final publication is available at

https://doi.org/10.1016/j.eswa.2015.05.058

Copyright Elsevier

Additional Information 


\title{
Methodology based on genetic heuristics for in-vivo characterizing the patient-specific biomechanical behavior of the breast tissues
}

\author{
M. A. Lago ${ }^{\mathrm{a}, *}$, M. J. Rúperez ${ }^{\mathrm{a}}$, F. Martínez-Martínez ${ }^{\mathrm{a}}$, P. R. Bakic ${ }^{\mathrm{b}}$, A. D. A. \\ Maidment $^{\mathrm{b}}$, C. Monserrat ${ }^{\mathrm{a}}$ \\ ${ }^{a}$ LabHuman, Universitat Politècnica de València, Camino de Vera s/n, 46022, Valencia, \\ Spain \\ ${ }^{b}$ Department of Radiology, University of Pennsylvania, Philadelphia PA, USA
}

\begin{abstract}
The accuracy of patient-specific biomechanical models of the breast is a major concern for applications such as surgical simulation, surgical guidance or cancer diagnosis. Being able to predict the localization of a lesion depends on the realism of the chosen model. However, the elastic parameters that define the biomechanical behavior of the breast tissues are highly variable among patients and their estimation becomes a very difficult task. This behavior is usually simulated with hyperelastic biomechanical models of the breast tissues. This paper presents an iterative search algorithm based on genetic heuristics which is able to estimate the elastic constants of a biomechanical model proposed to characterize the behavior of the breast tissues. Moreover, this methodology does not depend on the chosen biomechanical model. The algorithm was validated using breast software phantoms, compressed to mimic MRI-guided biopsies. The biomechanical model chosen to characterize the breast tissues was an anisotropic neo-Hookean hyperelastic model. Results from this analysis showed that the methodology is able to find the elastic constants of the constitutive equations of the proposed biomechanical model with a mean relative error of about $10 \%$. Keywords: genetic heuristics, in-vivo tissue characterization, breast
\end{abstract}

\footnotetext{
* Corresponding author

Email address: malago@labhuman.com (M. A. Lago)
} 
biomechanical modeling, parameter estimation

\section{Introduction}

The simulation of the mechanical behavior of the breast has become very relevant in the last years since it plays a main role in an important number of biomedical applications related to surgical simulations [1, 2, 3, 4], surgery guidance $[5,6]$ or cancer diagnosis $[7,8,9]$. These applications involve large deformations of the breast tissues such as mammographic compression or gravity loading deformation, which are usually modeled using the Finite Element Method (FEM).

One of the main challenges when modeling the biomechanical behavior of organs like the breast is to create patient-specific models that improve the realism and accuracy in a reasonable computation time. This is due to the high variability of the behavior of the breast tissues between patients and throughout the breast. However, the estimation of the biomechanical properties of the living tissues is not straightforward. The measurement of these properties is usually a complex task since the behavior of the tissues is highly variable between individuals. In the case of the breast, there are mainly three tissues whose behavior must be modeled, namely: skin, fat and glandular tissue. Each one of them has different biomechanical properties that must be estimated for each patient in order to build an accurate model of the whole breast.

Elastography is a common method for the in-vivo estimation of the elasticity of the breast $[10,11,12,13,14]$. This technique measures the dynamic stiffness of a tissue by cyclically applying a load. However, classic elastography is only useful to estimate the behavior of the tissues when they are considered isotropic and linearly elastic. Despite this limitation, use of elastography in the measuring of the viscoelasticity and hyperelasticity of the different breast tissues have been reported $[15,16]$.

In contrast, computational methods based on parameter optimization are being applied to characterize the biomechanical behavior of the in-vivo tissues. 
Specifically, evolutionary computation has been used in this field to identify the elastic constants of a hyperelastic model proposed to characterize the biomechanical behavior of the heart $[17,18]$ and also of the arterial wall [19]. In [20] our group presented a study of several evolutionary algorithms applied to in-vivo characterize the biomechanical behavior of the liver. The conclusion was that genetic heuristics performed better than other algorithms to estimate the elastic constants of an arbitrary biomechanical model proposed to simulate the liver behavior. The main advantages of this approach was the use of medical images that avoided the invasive measure of the mechanical response of the organ.

In the case of the breast, the work presented in [21] characterized the biomechanical behavior of the internal tissues of the breast in-vivo by means of an optimization algorithm which, using a compressed breast and measuring iteratively the similarity to a simulation of that deformation, provided the elastic constants of the proposed model. This is the first work in which the search was driven by a combination of a simulated annealing algorithm and a gradient descent algorithm in order to characterize the breast tissues. The authors used the Normalized Mutual Information (NMI) as a cost function to measure the similarity during the iterative search [22]. However, using this image-based comparison may result in inaccurate results since NMI does not consider the spatial distribution of the tissues but only the gray value entropy of both $3 \mathrm{D}$ images. In order to evaluate the accuracy of the given model, the cost function must consider the whole volume including the internal tissue distribution.

This work presents a methodology for estimating the in-vivo elastic constants specific to individual patients, of any biomechanical model proposed for characterizing the mechanical behavior of the breast internal tissues. A parameter optimization algorithm based on genetic heuristics and using volumetric comparison for evaluating the similarity was used to obtain a virtual deformed MRI of the breast as close as possible to a real deformed MRI. The methodology was validated using the software breast phantom proposed in [23] in order to speed up the calculations and mimic as much as possible the real breast tissue distribution. This methodology is easily applicable to real breast images and 
presents a novelty for the in-vivo characterization of the breast tissue mechanical behavior.

\section{Materials and Methods}

The methodology proposed in this paper is based on the acquisition by an MRI-guided biopsy device of two 3D images of the breast in different states of deformation. This device takes an MRI of the uncompressed breast in prone position as well as an MRI of the same breast under compression. The compression is performed by two rigid plates which hold the breast in a fixed position during the biopsy. The applied compression force must be known in order to perform the simulation of that compression. This force is provided by means of a force detector placed on the plates as described in [24]. From the MRI of the uncompressed breast, the simulation of the compression produced by the plates is performed using a biomechanical model proposed to emulate the behavior of the breast tissues. Then, an iterative search process is applied in order to find the elastic constants of the constitutive equations of the proposed model which provide the best fit between the simulated compressed MRI and the real compressed MRI.

In order to prove this methodology, breast software phantoms were used for creating synthetic cases similar to real ones while controlling all the constraints as well as reducing the amount of unknown boundary conditions. Since the biomechanical model needs the distribution of the different tissues of the breast, it is assumed that this segmentation has been already performed as in [4].

\subsection{Software phantom generation}

The breast phantoms used in this work were formed by three materials: fat tissue, glandular tissue and skin. The effect of the Cooper's ligaments was modeled by the anisotropy of the proposed biomechanical model [21]. The generation of the phantoms was carried out by recusrive partitioning using octrees and implemented on GPUs in order to speed up the process [25]. The breast phantoms consisted of a $3 \mathrm{D}$ raw volume simulating the distribution of fat and 


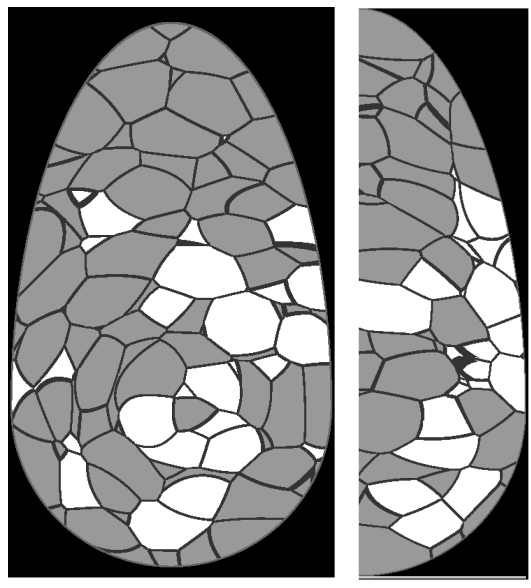

Figure 1: Left: coronal section of a raw phantom. Right: mediolateral section of the corresponding phantom. Each gray level denotes each tissue type: white pixels correspond to the glandular tissue, light gray pixels correspond to the fat tissue, dark gray lines correspond to the Cooper's ligaments and mid-dark gray pixels sourrounding the phantom correspond to the skin.

dense compartments in the breast volume separated by the Cooper's ligaments and wrapped by the skin. An example of a phantom is shown in Figure 1 [23].

\subsection{Biomechanical modeling}

Although most biomechanical models of the breast do not include the anisotropy of the Cooper's ligaments due to the difficulty of knowing their localization, some sensitivity studies considered that their influence is significant $[26,7,27]$. Furthermore, it must be considered that the breast is subjected to gravity loading in every acquisition technique due to the patient is in prone position. How to obtain the non-reference state of the breast, without loads, is something that is still under investigation [28]. Therefore, in order to model those influences in the behavior, the anisotropic hyperelastic model proposed in [21] was used in this work. The model proposed in [21] considers that the anisotropy due to the presence of Cooper's ligaments as well as the effect of the gravity force, can be modeled considering the breast as a fiber-reinforced material. They defined the orientation of the fibers in the chestwall-nipple direction which means that the breast is more likely to deform in the fiber direction. This 
fiber reinforcement allows to simulate the initial deformation of the breast due to the gravity force as well as considers the internal interactions of the Cooper's ligaments. The strain energy function for materials with fibers aligned in a specific direction can be defined as Eq. (1) shows, where the isotropic component and the fiber anisotropy are decomposed.

$$
W=W_{i s o}\left(I_{1}, I_{2}, I_{3}\right)+W_{f i b}\left(I_{4}\right)
$$

Following the indications in [21], a neo-Hookean hyperelastic model was chosen in order to reduce the number of variables of the model to be predicted. Eq. (2) shows the final energy function of the model used in this work.

$$
\begin{aligned}
W_{i s o}\left(I_{1}, I_{2}, I_{3}\right) & =\frac{\mu}{2}\left(I_{1}-3\right)+\frac{1}{d}(J-1)^{2} \\
W_{f i b}\left(I_{4}\right) & =\frac{\eta}{2}\left(I_{4}-1\right)^{2}
\end{aligned}
$$

where $\mu$ stands for the initial shear modulus of the material, $d$ stands for the incompressibility parameter of the material and $\eta$ stands for a parameter controlling the strength of the fibers.

Both $\mu$ and $d$ parameters can be determined from other two elastic parameters, the Young's modulus $E$ and the Poisson's ratio $\nu$ shown in Eq. (3).

$$
\begin{aligned}
& \mu=\frac{E}{2(1+\nu)} \\
& d=\frac{2}{k} \\
& k=\frac{E}{3(1-2 \nu)}
\end{aligned}
$$

The skin was considered isotropic with only one parameter to estimate, $E_{\text {skin. }}$. Assuming that all the tissues are incompressible $(\nu=0.49),\left\langle E_{f a t}\right.$, $\left.\eta_{f a t}, E_{\text {glandular }}, \eta_{\text {glandular }}, E_{\text {skin }}\right\rangle$ is the set of parameters to be estimated by the search algorithm. 


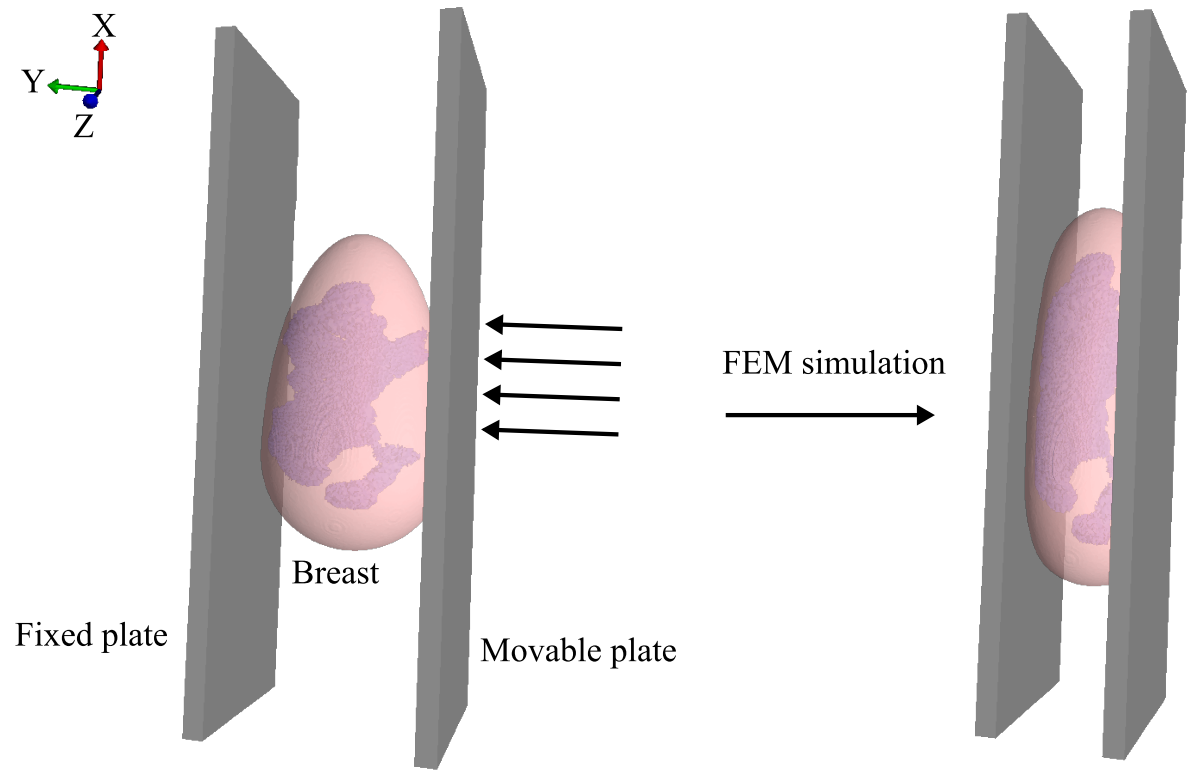

Figure 2: Simulation of the mammographic compression of a breast phantom.

\subsection{Boundary conditions and contact}

The mesh of the breast phantom was placed between two rigid plates, thus simulating the breast compression in an MRI biopsy device (Figure 2). Additionally, the corresponding nodes belonging to the chest wall were restricted in the chestwall-nipple direction $(\mathrm{Z})$ and some nodes already in contact with the plates were also restricted in the vertical direction $(\mathrm{X})$ to avoid rigid body displacement during the simulation. A force was applied to the moving plate in the $\mathrm{Y}$ direction while the other plate was completely fixed. To reduce the variability of the experiment and the number of variables affecting the whole simulation, the contact between the plates and the breast surface was modeled as a non-friction contact.

\subsection{Finite element mesh}

The finite element method was chosen to simulate the biomechanical behavior of the breast tissues under compression due to its ability to model complex geometries and boundary conditions. Usually, the finite element meshes that 
draw the boundary of the different tissues that forms an organ present convergence problems in the simulation of large deformations like the mammographic compression. This is mainly due to the bad quality of the generated elements.

In order to avoid this problem, the approach presented by our group in [29] was adopted to generate the FE meshes. In this approach, the meshes were generated with elements of similar size and shape, thus creating a more stable mesh which performs better under large deformations. The meshing algorithm is blind to the internal tissue distribution and generates a regular mesh with only one material. After the homogeneous mesh creation, each tetrahedron is assigned to the corresponding tissue: fat, glandular or skin. For that, gray values of the phantom at the tetrahedron vertices and at the centroid coordinates are extracted. Finally, each tetrahedron is assigned to the most common material from these 5 points.

\subsection{Volumetric similarity}

In order to evaluate the similarity of each virtual deformed breast with the real one accurately, the Geometric Similarity Function (GSF) [20, 30] was used in this work. This function is a combination of the Jaccard Coefficient [31] and the Modified Hausdorff Distance [32].

Jaccard Coefficient $J C$ measures the overlap between two volumes as Eq. (4) shows, where $V_{1}$ and $V_{2}$ stand for the volumes to be compared. $J C$ provides values between 0 and 1 , where 0 means no overlap and 1 means a total overlap.

$$
J C=\frac{V_{1} \cap V_{2}}{V_{1} \cup V_{2}}
$$

Modified Hausdorff Distance $M H D$ is defined in Eq. (5), $M H D$ measures the average distance between the voxel $i$ of a volume $V_{1}$ and the closest voxel of the other volume $V_{2}$.

$$
M H D=\max \left(\overline{d_{V_{1}}(i)}, \overline{d_{V_{2}}(i)}\right)
$$

$G S F$ is defined by the combination of $J C$ and $M H D$ as it is shown in Eq. 


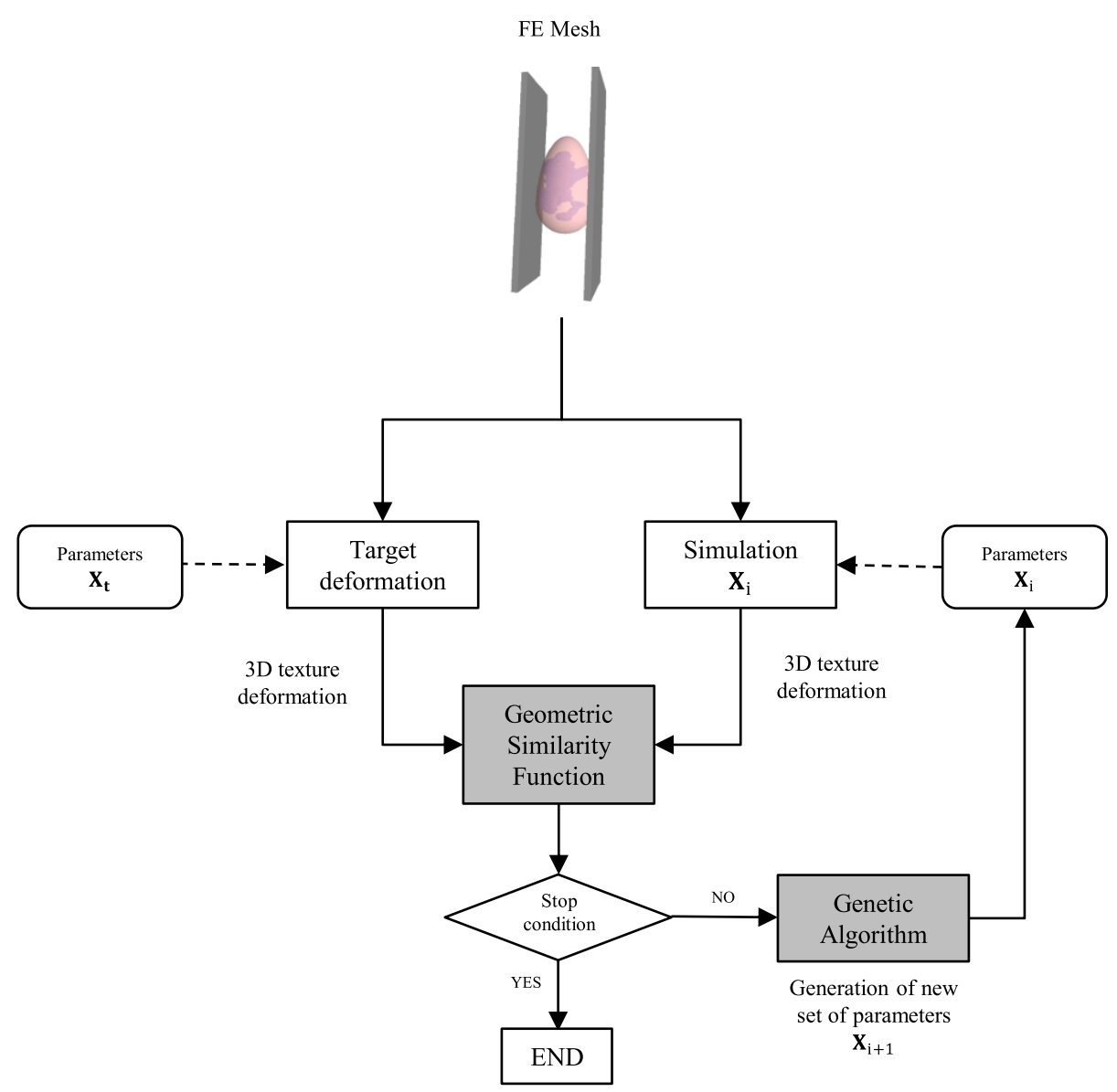

Figure 3: Flowchart of the optimization process using genetic algorithm.

160

(6). The lower the GSF values, the better similarity between volumes.

$$
G S F=\log ((1-J C) M H D)
$$

\subsection{Estimation of the Biomechanical Model}

A diagram of the iterative search algorithm is shown in Figure 3. First, the breast compression is simulated using the target set of parameters $\mathbf{X}_{\mathbf{t}}$. This simulation is used as a ground truth to evaluate the similarity of each candidate simulation during the iterative search.

Iterative search algorithms are often used to optimize a fit function $f(\mathbf{X})$, 
changing the input parameters $\mathbf{X}$ and using the output of the function to minimize or maximize its value.

$$
\hat{\mathbf{X}}=\arg \min f(\mathbf{X}) \quad \text { where } \mathbf{X}=\left\{x_{1}, x_{2}, \cdots, x_{n}\right\}
$$

However, in many applications, $f(\mathbf{X})$ usually has local minima that makes the simplest algorithms to get stuck, thus not being able to discover the global minimum of the function. For these cases, more complex algorithms like simulated annealing, scatter search or genetic algorithms must be implemented.

In [20], the capability of several evolutionary algorithms to estimate the elastic constants of the biomechanical models proposed for the liver was compared. As commented previously, the conclusion was that an iterative search based on genetic heuristics performed better for the estimation of these parameters. Therefore, in order to estimate the parameters of the considered breast tissues, a genetic algorithm was implemented in this work.

The outline of the implemented methodology is the following:

1. Initialize: a random population of samples $\mathbf{X}_{0}$ is created. It is common to set an interval for each parameter to be found in order to help the algorithm to search in the area where the global minimum of the function may be located.

2. New population generation: iteratively, the algorithm creates a new candidate set of parameters $\mathbf{X}_{i+1}$ by means of the following steps:

a) The algorithm computes $f(x)$ for each individual in the current set $\mathbf{X}_{i}$.

b) Those individuals with the best score are selected as parents.

c) Parents with the best score are tagged as elite and pass directly to the next population.

d) Non-elite parents are used to generate new children both by mutation (randomly changing a parent) and by crossover (combination of several parents).

e) The next candidate population $\mathbf{X}_{i+1}$ is created by joining elite and children. 
3. Termination: step 2 is repeated until a stop condition is reached. This can be a specific number of generations, a timer, or when the function does not change within a tolerance range. Finally, the set of parameters that minimized the function is designated as $\hat{\mathbf{X}}_{\mathbf{t}}$.

In each generation $i$ of the algorithm, the candidate sets of parameters $\mathbf{X}_{i}$ are applied to the model to simulate the breast compression. Both the target deformation and the candidate simulation are used to deform the 3D software phantom, thus having a target phantom and a candidate phantom. The creation of the deformed software phantoms was carried out on the GPU, considering the undeformed phantom as a 3D texture and using a linear interpolation of the gray levels over each deformed element of the mesh. The comparison was carried out only using the glandular tissue compartments with the GSF as fit function. The larger size of fat tissue with regard to glandular tissue could cause the average values of GSF to be less significant. Additionally, the main differences were located in the neighborhood of glandular tissue compartments. Therefore the focus was made on those areas.

Finally, the stop condition is evaluated. In the case of not achieving a low enough value of GSF, the genetic algorithm takes over the task of generating a new set of parameters $\mathbf{X}_{i+1}$ and the iterative process starts again until an optimum value of the GSF is obtained.

The iterative search was developed in a MATLAB script using the genetic algorithm implemented in this software and accessible using the function $g a$ [33]. Taking advantage of the independent simulations of the genetic algorithm within the same generation, the process was parallelized in the different cores of the computer thus accelerating the search.

\section{Results}

Ten phantoms with glandular density between $7 \%$ and $35 \%$, with a volume of $450 \mathrm{ml}$ and identical shape were generated. For all of them, the size of the uncompressed phantoms was $17 \mathrm{~cm}$ in vertical direction, $10 \mathrm{~cm}$ in lateral direction 
and $5 \mathrm{~cm}$ in chestwall-nipple direction. Resolution of the phantom voxel was set to be $200 \mu m$, which was small enough to detect the slightest differences between candidate and target deformations.

A uniformly distributed force of $100 N$ was applied on the movable plate. This value was chosen as the average value of the forces applied to perform mammographic compression to real patients during X-ray mammography [4].

The experiment considered three different sets of target parameters. For a first validation of the methodology, in the two first experiments, $\mathbf{X}_{\mathbf{t}}^{\mathbf{1}}$ and $\mathbf{X}_{\mathbf{t}}^{\mathbf{2}}$, the skin tissue was not considered and was treated as fat tissue. These two experiments allowed to simplify the model. A third experiment was then carried out, this time taking into account the skin, thus having a complete model of the breast $\mathbf{X}_{\mathbf{t}}^{3}$.

Target and predicted parameters for each one of the phantoms are shown in Tables 1, 2 and 3. It is important to notice that although $G S F$ is very useful to discriminate good and bad volume similarity, there is no natural interpretation of its values. Therefore, the tables show the values of both $J C$ and $M H D$ for interpretation purposes.

Considering the variability of the biomechanical behavior of glandular and fat tissues estimated by [21], the search space of the iterative algorithm was defined by the following initial intervals:

$$
\begin{aligned}
E_{f a t} & \in[5000-20000] \mathrm{Pa} \\
\eta_{\text {fat }} & \in[50000-200000] \\
E_{\text {glandular }} & \in[5000-80000] \mathrm{Pa} \\
\eta_{\text {glandular }} & \in[50000-200000] \\
E_{\text {skin }} & \in[200000-3000000] \mathrm{Pa}
\end{aligned}
$$

The genetic algorithm configuration was set up as follows: the population size for each iteration was set to 84 in order to paralellize the process among the 12 cores of the computer. The crossover fraction was set to 0.8 , this meant that 
Table 1: Parameters for the target deformation $\mathbf{X}_{\mathbf{t}}^{\mathbf{1}}$ and estimated parameters for the model without skin.

\begin{tabular}{l|cc|cc|cc}
\hline & $\begin{array}{c}E_{f a t} \\
(\mathrm{~Pa})\end{array}$ & $\eta_{\text {fat }}$ & $\begin{array}{c}E_{\text {glandular }} \\
(\mathrm{Pa})\end{array}$ & $\eta_{\text {glandular }}$ & $J C$ & $\begin{array}{c}\text { MHD } \\
(\mathrm{vox})\end{array}$ \\
\hline $\mathbf{X}_{\mathbf{t}}^{\mathbf{1}}$ & $\mathbf{1 0 ~ 0 0 0}$ & $\mathbf{1 0 0} \mathbf{0 0 0}$ & $\mathbf{4 0 ~ 0 0 0}$ & $\mathbf{1 5 0 ~ 0 0 0}$ & 1 & 0 \\
\hline$\hat{\mathbf{X}}_{\mathbf{t}}^{\mathbf{1}}$ Phantom 1 & 9746 & 107720 & 49812 & 119410 & 0.947 & 0.689 \\
$\hat{\mathbf{X}}_{\mathbf{t}}^{\mathbf{1}}$ Phantom 2 & 10036 & 104840 & 40049 & 126520 & 0.988 & 0.20 \\
$\hat{\mathbf{X}}_{\mathbf{t}}^{\mathbf{1}}$ Phantom 3 & 9766 & 119430 & 47541 & 114900 & 0.944 & 0.788 \\
$\hat{\mathbf{X}}_{\mathbf{t}}^{\mathbf{1}}$ Phantom 4 & 10086 & 113560 & 37552 & 110840 & 0.978 & 0.422 \\
$\hat{\mathbf{X}}_{\mathbf{t}}^{\mathbf{1}}$ Phantom 5 & 10303 & 91353 & 40256 & 60956 & 0.913 & 0.90 \\
\hline Avg. $\hat{\mathbf{X}}_{\mathbf{t}}^{\mathbf{1}}$ & 9987 & 107381 & 43042 & 106525 & - & - \\
Std. Dev. & 234 & 10569 & 5314 & 26130 & - & - \\
\hline Error & $1.83 \%$ & $10.84 \%$ & $10.05 \%$ & $28.98 \%$ & - & - \\
\hline
\end{tabular}

the $80 \%$ of the children were generated by mutation and the $20 \%$ by crossover; the elite count was set to 2, these are default values in MATLAB. Finally, the number of generations was set to 15 , ensuring enough exploration of the search space in a reasonable computation time. This configuration provided good results previously [20]. These parameters can be tuned for each problem and the results may improve, a specific study for each patient could be performed in order to know the best configuration for the genetic algorithm.

The commercial FE package ANSYS ${ }^{\circledR}$ was used to simulate the target deformation as well as each candidate simulation. The glandular compartments of the candidate compressed phantoms were compared with the same compartments of the target compressed phantom using $G S F$ in a parallelized MATLAB script. The number of simulations needed to achieve the final values varied between phantoms and was about 1000 simulations in $48 \mathrm{~h}$ of computation time. The used computer was an Intel Xeon X5650@2.66 GHz (12 cores) with 64GB of RAM.

Figure 4 shows one section of the same phantom deformed using the target parameters (left) and the estimated parameters (middle). Additionally, the right image shows their absolute differences, white pixels denote the non matching 
Table 2: Parameters for the target deformation $\mathbf{X}_{\mathbf{t}}^{\mathbf{2}}$ and estimated parameters for the model without skin.

\begin{tabular}{l|cc|cc|cc}
\hline & $\begin{array}{c}E_{\text {fat }} \\
(\mathrm{Pa})\end{array}$ & $\eta_{\text {fat }}$ & $\begin{array}{c}E_{\text {glandular }} \\
(\mathrm{Pa})\end{array}$ & $\eta_{\text {glandular }}$ & $J C$ & $\begin{array}{c}M H D \\
\text { (vox) }\end{array}$ \\
\hline $\mathbf{X}_{\mathbf{t}}^{\mathbf{2}}$ & $\mathbf{7 5 0 0}$ & $\mathbf{7 5} \mathbf{0 0 0}$ & $\mathbf{3 0 ~ 0 0 0}$ & $\mathbf{1 1 2} \mathbf{5 0 0}$ & 1 & 0 \\
\hline$\hat{\mathbf{X}}_{\mathbf{t}}^{\mathbf{2}}$ Phantom 6 & 7538 & 73112 & 29826 & 121820 & 0.991 & 0.226 \\
$\hat{\mathbf{X}}_{\mathbf{t}}^{\mathbf{2}}$ Phantom 7 & 6785 & 96682 & 31488 & 154810 & 0.926 & 0.667 \\
$\hat{\mathbf{X}}_{\mathbf{t}}^{2}$ Phantom 8 & 7523 & 95324 & 28292 & 74674 & 0.953 & 0.652 \\
$\hat{\mathbf{X}}_{\mathbf{t}}^{\mathbf{2}}$ Phantom 9 & 6520 & 75593 & 34445 & 180770 & 0.923 & 0.850 \\
$\hat{\mathbf{X}}_{\mathbf{t}}^{2}$ Phantom 10 & 7532 & 71527 & 29717 & 99797 & 0.988 & 0.258 \\
\hline Avg. $\hat{\mathbf{X}}_{\mathbf{t}}^{\mathbf{2}}$ & 7180 & 82448 & 30754 & 126374 & - & - \\
Std. Dev. & 490 & 12468 & 2353 & 42330 & - & - \\
\hline Error & $4.77 \%$ & $12.79 \%$ & $5.40 \%$ & $30.30 \%$ & - & - \\
\hline
\end{tabular}

Table 3: Parameters for the target deformation $\mathbf{X}_{\mathbf{t}}^{\mathbf{3}}$ and estimated parameters for the model considering the skin.

\begin{tabular}{|c|c|c|c|c|c|c|c|}
\hline & $\begin{array}{l}E_{f a t} \\
(\mathrm{~Pa})\end{array}$ & $\eta_{f a t}$ & $\begin{array}{c}E_{\text {glandular }} \\
(\mathrm{Pa})\end{array}$ & $\eta_{\text {glandular }}$ & $\begin{array}{c}E_{\text {skin }} \\
(\mathrm{Pa})\end{array}$ & $J C$ & $\begin{array}{c}M H D \\
(\operatorname{vox})\end{array}$ \\
\hline$X_{t}^{3}$ & 10000 & 100000 & 40000 & 150000 & 1600000 & 1 & 0 \\
\hline$\hat{\mathbf{X}}_{\mathbf{t}}^{\mathbf{3}} \mathrm{Ph} .1$ & 10086 & 101290 & 37390 & 160110 & 1577800 & 0.933 & 0.72 \\
\hline$\hat{\mathbf{X}}_{\mathbf{t}}^{\mathbf{3}}$ Ph. 2 & 10116 & 102534 & 69040 & 159300 & 1492338 & 0.91 & 2.29 \\
\hline$\hat{\mathbf{X}}_{\mathrm{t}}^{\mathbf{3}} \mathrm{Ph} .3$ & 9886 & 84556 & 40958 & 87594 & 1637200 & 0.961 & 1.71 \\
\hline$\hat{\mathbf{X}}_{\mathbf{t}}^{\mathbf{3}} \mathrm{Ph} .4$ & 11372 & 87682 & 30150 & 165830 & 1502500 & 0.949 & 1.18 \\
\hline$\hat{\mathbf{X}}_{\mathrm{t}}^{\mathbf{3}} \mathrm{Ph} .5$ & 11452 & 77835 & 40307 & 191230 & 1499500 & 0.90 & 1.29 \\
\hline Avg. $\hat{\mathbf{X}}_{\mathbf{t}}^{\mathbf{3}}$ & 10369 & 92845 & 37817 & 155159 & 1572020 & - & - \\
\hline Std. Dev. & 1029 & 14076 & 4503 & 39549 & 69689 & - & - \\
\hline Error & $7.95 \%$ & $12.82 \%$ & $7.00 \%$ & $20.08 \%$ & $4.56 \%$ & - & - \\
\hline
\end{tabular}




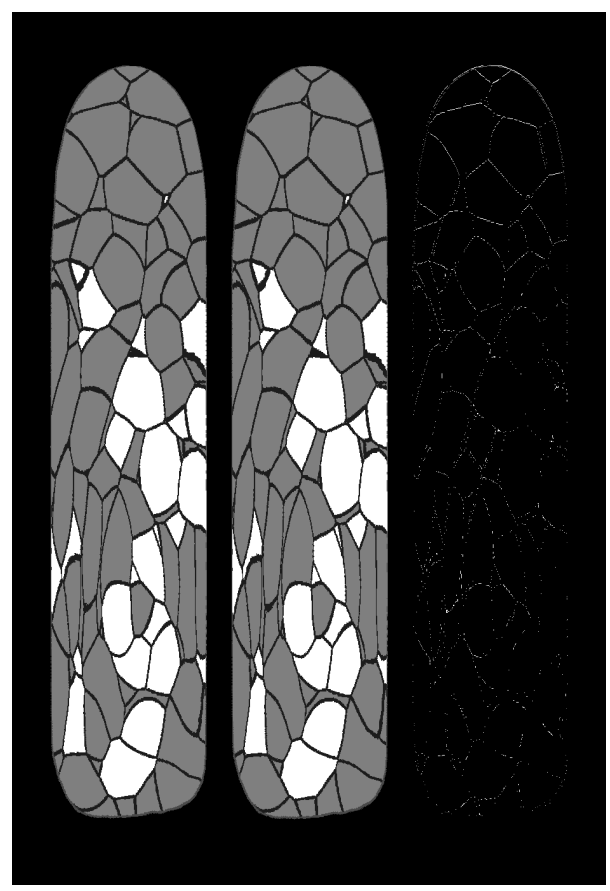

Figure 4: Left: Coronal section of the deformed phantom using the target parameters $\mathbf{X}_{\mathbf{t}}^{\mathbf{1}}$. Middle: Coronal section of the deformed phantom with the estimated parameters. Right: Difference between target and estimated deformed phantoms. In the right image, white pixels correspond to mismatching voxels. 
pixels between the target and estimated deformed phantom.

\section{Discussion}

The first two experiments achieved a mean relative error of $1.83 \%$ and $4.77 \%$ for $E_{f a t}, 10.05 \%$ and $5.40 \%$ for $E_{\text {glandular }}$ and $10.84 \%$ and $12.79 \%$ for $\eta_{f a t}$. These errors are relatively low and the estimation of these parameters with the presented methodology can be considered successful. Regarding the parameter controlling the fiber strength for the glandular tissue, $\eta_{\text {glandular }}$, its estimation was not so accurate.

To analyze this result, a sensibility analysis was performed in order to know the influence of this parameter in the model. To perform this, all the parameters except $\eta_{\text {glandular }}$ were fixed to their target values. Then, $\eta_{\text {glandular }}$ was iterated separately over the search interval [50000 - 200000] and the deformed phantom obtained with this set of parameters was compared to the target phantom. Figure 5 shows a graph with the tendency of $J C$ and $M H D$ when varying $\eta_{\text {glandular }}$ over the initial search interval. Values of $J C>0.93$ and $M H D<1$ voxels in the whole range proved the low influence of this parameter in the model.

The $\eta$ parameters take into account two effects: gravity force and influence of Cooper's ligaments. On one hand, the breast is subjected to initial strainsstresses due to the gravity force in both states, compressed and uncompressed. Ideally, the deformation caused by the gravity force must be considered separately of the tissue deformation model. Unfortunately, knowing the non-strain state of the breast is something that is still being investigated [28]. On the other hand, the influence of the Cooper's ligaments was modeled only in one direction as stated in [6]. Since they have an unknown effect on the model the effect of these ligaments could be modeled in the three directions of the space. This would involve that new parameters should be added to the model. Nevertheless, they could also be estimated with the proposed methodology.

Regarding the anisotropic parameter for the fat tissue, $\eta_{f a t}$, its estimation was more accurate with an error lower than $13 \%$. This discrepancy with the 
estimation of $\eta_{\text {glandular }}$ can be explained due to the higher presence of fat tissue in the breast as well as the higher influence of the Cooper's ligaments in this region. This results in a higher effect of $\eta_{f a t}$ on the model compared to the effect of $\eta_{\text {glandular }}$.

It is important to highlight the importance of $J C$ and $M H D$ which indicate how much accurate the estimation was. The best estimated set of parameters were for Phantoms \#2 and \#6, which $J C$ values were about 0.99 and $M H D$ was 0.2 voxels $(1$ vox $=200 \mu \mathrm{m})$. These are good indicators of the accuracy of the parameter estimation which, especially in these cases, were estimated very close to the target parameters with errors lower than $1 \%$ for $E$ and lower than $5 \%$ for $\eta_{f a t}$. Other phantoms with worse values of these coefficients were estimated less accurately. However, modifying the initial setup of the genetic algorithm could improve those values.

As for the estimation of the whole model of the breast, including the skin, the accuracy of the elastic parameters showed errors lower than $8 \%$. The addition of the skin to the model did not decrease the performance of the methodology. In this case, the estimated elasticity for the skin was achieved with a $4.56 \%$ of relative mean error which indicates a high influence in the breast model as reported in [4]. In contrast, the estimation of the $\eta$ parameters showed an accuracy in consonance with the first two experiments, where $\eta_{\text {glandular }}$ did not induce much variability within the search range.

The number of elements of the biomechanical model also influenced the search algorithm. Increasing the element density would impact highly the time needed to solve the contact problem but would also increase the accuracy of the search. Furthermore, reducing the search intervals would cause the algorithm to converge faster by reducing the search space. In this paper, those intervals were set particularly wide in order to prove the suitability of the methodology in case of barely knowing the elastic parameters of the different tissues. Increasing the complexity of the problem by using a biomechanical model with more parameters would cause the algorithm to converge slower. Nevertheless, the methodology could still be applied since genetic heuristics are very efficient 


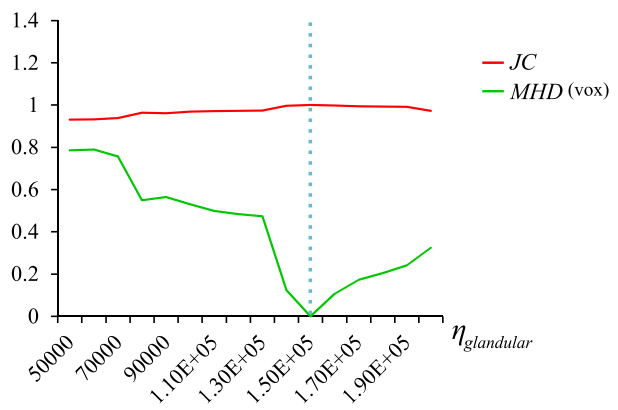

Figure 5: Sensitivity test over the glandular tissue. $J C$ and $M H D$ in terms of $\eta_{\text {glandular }}$. The dotted line is the corresponding value to the target phantom.

when having a problem with many variables to optimize [20].

The application of the methodology to real breasts is straightforward. Despite the higher complexity of the internal distribution of the breast tissues, the MRI can be segmented and the comparison between the real compressed MRI and each candidate biomechanical model can follow the same procedure.

\section{Conclusion}

The methodology described in this paper allows to in-vivo estimate the patient-specific biomechanical properties of the breast tissues. The different tissues of the breast were this way characterized, providing the elastic constants of an anisotropic hyperelastic model for the fat and glandular tissues and for an isotropic elastic model in the case of the skin. The genetic algorithm was able to find a set of elastic parameters almost identical to the target ones without knowing anything about the original behavior and in a wide search space. The performance of the methodology was proved with breast phantoms achieving an estimation error of less than $10 \%$. This methodology can be easily applied to characterize the biomechanical model for real breasts.

Our ongoing research is the application of the proposed methodology to real breasts. Future works will include the characterization of a complete model for the breast able to simulate the deformation that the breast undergoes during X-ray mammography and the tuning of the initial setup of the genetic algorithm for each patient. 


\section{Acknowledgements}

This project has been funded by MECD (reference AP2009-2414) and US National Institutes of Health (R01 grant \#CA154444), and the US National Science Foundation (III grant \#0916690). The content is solely the responsibility of the authors and does not necessarily represent the official views of the NIH, and NSF. The authors of this manuscript have no conflict of interest with the presented work.

\section{References}

[1] C. Tanner, T. Carter, H. D.J., 3D Rezoning for Finite Element Modelling of Large Breast Deformations, in: Proceedings of European Modelling Symposium, 2006, pp. 51-53.

[2] A. P. del Palomar, B. Calvo, J. Herrero, J. López, M. Doblaré, A finite element model to accurately predict real deformations of the breast, Medical Engineering and Physics 30 (9) (2008) 1089 - 1097. doi:http://dx.doi.org/10.1016/j.medengphy. 2008.01.005.

URL http://www.sciencedirect.com/science/article/pii/ S1350453308000210

[3] C. M. L. Hsu, M. L. Palmeri, W. P. Segars, A. I. Veress, J. T. D. III, An analysis of the mechanical parameters used for finite element compression of a high-resolution 3D breast phantom, Medical Physics 38 (10) (2011) 5756-5770. doi:10.1118/1.3637500.

[4] J. Solves Llorens, C. Monserrat, M. Rupérez Moreno, V. Naranjo, M. Alajami, E. Feliu, M. García, M. Lloret, MRI Skin Segmentation for the Virtual Deformation of the Breast under Mammographic Compression, in: MMVR19, 2012, pp. 483-489.

[5] T. Carter, C. Tanner, N. Beechey-Newman, D. Barratt, D. Hawkes, MR Navigated Breast Surgery: Method and Initial Clinical Experience, 
in: Proceedings of the 11th International Conference on Medical Image Computing and Computer-Assisted Intervention, Part II, MICCAI '08, Springer-Verlag, Berlin, Heidelberg, 2008, pp. 356-363. doi:10.1007/ 978-3-540-85990-1\_43.

URL http://dx.doi.org/10.1007/978-3-540-85990-1_43

[6] L. Han, J. Hipwell, T. Mertzanidou, T. Carter, M. Modat, S. Ourselin, D. Hawkes, A hybrid fem-based method for aligning prone and supine images for image guided breast surgery, in: Biomedical Imaging: From Nano to Macro, 2011 IEEE International Symposium on, 2011, pp. 1239-1242. doi:10.1109/ISBI.2011.5872626.

[7] N. Ruiter, R. Stotzka, T.-O. Muller, H. Gemmeke, J. Reichenbach, W. Kaiser, Model-based registration of X-ray mammograms and MR images of the female breast, Nuclear Science, IEEE Transactions on 53 (1) (2006) 204-211. doi:10.1109/TNS.2005.862983.

[8] P. Pathmanathan, D. Gavaghan, J. Whiteley, S. Chapman, J. Brady, Predicting Tumor Location by Modeling the Deformation of the Breast, Biomedical Engineering, IEEE Transactions on 55 (10) (2008) 2471-2480. doi:10.1109/TBME. 2008.925714.

[9] V. Rajagopal, P. M. F. Nielsen, M. P. Nash, Modeling breast biomechanics for multi-modal image analysis - successes and challenges, Wiley Interdisciplinary Reviews: Systems Biology and Medicine 2 (3) (2010) 293-304. doi:10.1002/wsbm. 58.

URL http://dx.doi.org/10.1002/wsbm.58

[10] J. Ophir, I. Cspedes, H. Ponnekanti, Y. Yazdi, X. Li, Elastography: A quantitative method for imaging the elasticity of biological tissues, Ultrasonic Imaging 13 (2) (1991) 111 - 134. doi:http://dx.doi .org/10.1016/0161-7346(91)90079-W. URL http://www.sciencedirect.com/science/article/pii/ 016173469190079W 
[11] T. A. Krouskop, T. M. Wheeler, F. Kallel, B. S. Garra, T. Hall, Elastic moduli of breast and prostate tissues under compression, Ultrasonic Imaging 20 (4) (1998) 260-274. arXiv:http://uix. sagepub.com/content/20/ 4/260.full.pdf+html, doi:10.1177/016173469802000403.

URL http://uix.sagepub.com/content/20/4/260. abstract

[12] J. F. Greenleaf, M. Fatemi, M. Insana, Selected methods for imaging elastic properties of biological tissues, Annual Review of Biomedical Engineering 5 (1) (2003) 57-78, pMID: 12704084. arXiv:http: //www. annualreviews.org/doi/pdf/10.1146/annurev. bioeng. 5 . 040202.121623, doi:10.1146/annurev.bioeng.5.040202.121623. URL http://www. annualreviews.org/doi/abs/10.1146/annurev . bioeng.5.040202.121623

[13] Y. K. Mariappan, K. J. Glaser, R. L. Ehman, Magnetic resonance elastography: A review, Clinical Anatomy 23 (5) (2010) 497-511. doi: $10.1002 / \mathrm{ca} .21006$.

URL http://dx.doi.org/10.1002/ca.21006

[14] R. G. Barr, Sonographic breast elastography: A primer, Journal of Ultrasound in Medicine 31 (5) (2012) 773-783. arXiv:http://www. jultrasoundmed.org/content/31/5/773.full.pdf+html.

[15] R. Sinkus, M. Tanter, T. Xydeas, S. Catheline, J. Bercoff, M. Fink, Viscoelastic shear properties of in vivo breast lesions measured by MR elastography, Magnetic Resonance Imaging 23 (2) (2005) 159 - 165. doi:http://dx.doi.org/10.1016/j.mri.2004.11.060.

URL http://www.sciencedirect.com/science/article/pii/ S0730725X05000391

[16] H. Mehrabian, G. Campbell, A. Samani, A constrained reconstruction technique of hyperelasticity parameters for breast cancer assessment, Physics in Medicine and Biology 55 (24) (2010) 7489.

URL http://stacks . iop.org/0031-9155/55/i=24/a=007 
[17] A. Pandit, X. Lu, C. Wang, G. S. Kassab, Biaxial elastic material properties of porcine coronary media and adventitia, American Journal of Physiology - Heart and Circulatory Physiology 288 (6) (2005) H2581-H2587. arXiv:http://ajpheart . physiology . org/content/288/ 6/H2581.full.pdf, doi:10.1152/ajpheart.00648. 2004. URL http://ajpheart.physiology.org/content/288/6/H2581

[18] A. U. Nair, D. G. Taggart, F. J. Vetter, Optimizing cardiac material parameters with a genetic algorithm, Journal of Biomechanics 40 (7) (2007) 1646 -1650. doi:http://dx.doi.org/10.1016/j.jbiomech.2006.07.018.

URL http://www.sciencedirect.com/science/article/pii/ S0021929006002764

[19] N. Harb, N. Labed, M. Domaszewski, F. Peyraut, A new parameter identification method of soft biological tissue combining genetic algorithm with analytical optimization, Computer Methods in Applied Mechanics and Engineering 200 (14) (2011) 208 - 215. doi:http://dx.doi.org/10.1016/j.cma.2010.08.005.

URL http://www.sciencedirect.com/science/article/pii/ S0045782510002380

[20] F. Martínez-Martínez, M. Rupérez, J. Martín-Guerrero, C. Monserrat, M. Lago, E. Pareja, S. Brugger, R. López-Andújar, Estimation of the elastic parameters of human liver biomechanical models by means of medical images and evolutionary computation, Computer Methods and Programs in Biomedicine 111 (3) (2013) 537 - 549. doi:http://dx.doi.org/10. $1016 / j \cdot c m p b .2013 .05 .005$.

[21] L. Han, J. H. Hipwell, C. Tanner, Z. Taylor, T. Mertzanidou, J. Cardoso, S. Ourselin, D. J. Hawkes, Development of patient-specific biomechanical models for predicting large breast deformation, Physics in Medicine and Biology 57 (2) (2012) 455.

URL http://stacks.iop.org/0031-9155/57/i=2/a=455 
[22] C. Studholme, D. Hill, D. Hawkes, An overlap invariant entropy measure of 3d medical image alignment, Pattern Recognition 32 (1) (1999) 71 - 86. doi:http://dx.doi.org/10.1016/S0031-3203(98)00091-0. URL http://www.sciencedirect.com/science/article/pii/ S0031320398000910

[23] D. D. Pokrajac, A. D. A. Maidment, P. R. Bakic, Optimized generation of high resolution breast anthropomorphic software phantoms, Medical Physics 39 (4) (2012) 2290-2302. doi:10.1118/1.3697523.

[24] U.-X. Tan, B. Yang, R. Gullapalli, J. Desai, Design and development of a 3-axis mri-compatible force sensor, in: Robotics and Automation (ICRA), 2010 IEEE International Conference on, 2010, pp. 2586-2591. doi:10. 1109/ROBOT. 2010.5509761.

[25] J. H. Chui, D. D. Pokrajac, A. D. A. Maidment, P. R. Bakic, Towards Breast Anatomy Simulation Using GPUs, in: Digital Mammography / IWDM, 2012, pp. 506-513.

[26] C. Tanner, J. A. Schnabel, D. L. G. Hill, D. J. Hawkes, M. O. Leach, D. R. Hose, Factors influencing the accuracy of biomechanical breast models, Medical Physics 33 (6) (2006) 1758-1769. doi:http://dx.doi.org/10. $1118 / 1.2198315$.

URL http://scitation.aip.org/content/aapm/journal/medphys/33/ $6 / 10.1118 / 1.2198315$

[27] A. Gefen, B. Dilmoney, Mechanics of the normal women breast, Technol. Health Care 15 (4) (2007) 259-271.

[28] V. Rajagopal, A. Lee, J.-H. Chung, R. Warren, R. P. Highnam, M. P. Nash, P. M. Nielsen, Creating Individual-specific Biomechanical Models of the Breast for Medical Image Analysis, Academic Radiology 15 (11) (2008) 1425 - 1436. doi:http://dx.doi.org/10.1016/j . acra. 2008.07.017. URL http://www.sciencedirect.com/science/article/pii/ S1076633208004224 
[29] M. Lago, M. Rupérez, C. Monserrat, Impact of the meshing method in the biomechanical simulation of the mammographic compression, in: Proceedings of III Reunión del Capítulo Nacional Español de la SOCIEDAD EUROPEA de BIOMECÁNICA, Institut de Bioenginyeria de Catalunya, 2013, p. 38.

[30] F. Martínez-Martínez, M. J. Rupérez, J. D. Martín-Guerrero, C. Monserrat, E. Pareja, S. Brugger, R. López-Andújar, Estimation of the biomechanical parameters of the human liver avoiding invasive measuring methods, in: Proc. of the 19th Congress of the European Society of Biomechanics (ESB 2013), 2013, p. 537549.

[31] P. Jaccard, Étude comparative de la distribution florale dans une portion des Alpes et des Jura, Bulletin del la Société Vaudoise des Sciences Naturelles 37 (1901) 547-579.

[32] M. P. Dubuisson, A. K. Jain, A modified hausdorff distance for object matching, in: Proceedings of 12th International Conference on Pattern Recognition, Vol. 1, IEEE Comput. Soc. Press, 1994, pp. 566-568.

[33] A. Chipperfield, P. Fleming, The matlab genetic algorithm toolbox, in: Applied Control Techniques Using MATLAB, IEE Colloquium on, 1995, pp. 10/1-10/4. doi:10.1049/ic:19950061. 